Original Article

\title{
Comparison of treatment effect of neuromuscular electrical stimulation and thermal-tactile stimulation on patients with sub-acute dysphagia caused by stroke
}

\author{
Haewon Byeon, DrSc ${ }^{1)}$, Hyeung Woo Koh, PT, $\left.\mathrm{PhD}^{2}\right)^{*}$ \\ 1) Department of Speech Language Pathology, Nambu University, Republic of Korea \\ 2) Department of Physical Therapy, Jeju Seogwipo Medical Center: Jeju, Republic of Korea
}

\begin{abstract}
Purpose] The effectiveness of neuromuscular electrical stimulation in the rehabilitation of swallowing remains controversial. This study compared the effectiveness of neuromuscular electrical stimulation and thermal tactile oral stimulation, a traditional swallowing recovery treatment, in patients with sub-acute dysphagia caused by stroke. [Subjects and Methods] Subjects of the present study were 55 patients diagnosed with dysphagia caused by stroke. This study had a nonequivalent control group pretest-posttest design. [Results] Analysis of pre-post values of videofluoroscopic studies of the neuromuscular electrical stimulation and thermal tactile oral stimulation groups using a paired t-test showed no significant difference between the two groups despite both having decreased mean values of the videofluoroscopic studies after treatment. [Conclusion] This study's findings show that both neuromuscular electrical stimulation and thermal tactile oral stimulation significantly enhanced the swallowing function of patients with sub-acute dysphagia.

Key words: Neuromuscular electrical stimulation, Thermal tactile oral stimulation, Dysphagia
\end{abstract}

(This article was submitted Jan. 19, 2016, and was accepted Mar. 12, 2016)

\section{INTRODUCTION}

Swallowing is a series of coordinated processes in which food boluses move from the mouth to the stomach. Healthy people generally do not need any special effort to swallow food. The normal process of swallowing includes the oral preparatory phase in which food is chewed; the oral phase in which food is moved to the rear of the oral cavity and swallowing starts; the pharyngeal phase in which food is quickly moved through the pharynx to the esophagus; and the esophageal phase in which food is moved down the esophagus to the stomach ${ }^{1)}$. If problems exist in the nerves or muscles involved in this process, a person cannot swallow food safely. Thus, it is important to determine the causes of swallowing problems by stage and provide proper treatment to help patients with dysphagia recover their swallowing function.

The pharyngeal phase in particular is involuntary, and since the airway and esophagus are closely located each other, it is very important that the pharyngeal muscles operate normally to prevent food boluses from entering the airway. If problems occur with coordination of the swallowing muscles in the pharyngeal stage, food can get through the airway and cause serious complications such as suffocation or aspiration pneumonia. This kind of swallowing dysfunction is called dysphagia. Dysphagia can be caused by various diseases that can affect the nerves or muscles; swallowing problems in the pharyngeal phase in particular are frequently caused by damage to the neurological system such as stroke ${ }^{1)}$. Dysphagia affects a reported $40-70 \%$ of acute stroke patients ${ }^{2,3)}$ and becomes a direct cause of malnutrition, dehydration, and pneumonia. In particular, since stroke patients are at high risk of aspiration or penetration, both of which involve the passage of food into the airway

*Corresponding author. Hyeung Woo Koh (E-mail: kohw0925@naver.com)

(C2016 The Society of Physical Therapy Science. Published by IPEC Inc.

This is an open-access article distributed under the terms of the Creative Commons Attribution Non-Commercial No Derivatives (by-nc-nd) License $<$ http://creativecommons.org/licenses/by-nc-nd/4.0/>. 
due to swallowing problems in the pharyngeal phase, it is critical that they obtain swallowing treatments as soon as dysphagia is discovered ${ }^{4}$.

Treatment strategies for dysphagia in the oral cavity and pharynx are largely divided into feeding techniques and swallowing therapy, the latter of which induces swallowing and enhances the pharyngeal phase. Of these two strategies, swallowing therapy includes techniques to prolong pharyngeal swallowing, enhances pharyngeal transit time, and facilitates neuromuscular activities related to swallowing; traditionally, it includes compensatory approaches such as posture changes and maneuvers that improve function by strengthening weak swallowing muscles ${ }^{1}$.

Neuromuscular electrical stimulation (NMES), which induces the functional recovery of swallowing using electrical stimulation on paralyzed cervical muscles, is frequently used in swallowing treatment. A non-invasive treatment, NMES has the advantage of being easier to apply than traditional treatment techniques such as compensatory approach and can be effectively used for patients with damaged cognitive function for whom compensatory treatment is difficult to apply ${ }^{5}$.

Nevertheless, the effectiveness of NMES in the rehabilitation of swallowing remains controversial. While there are reports that NMES is significantly more effective in treatment than tactile stimulation treatment or compensatory treatment techniques $^{5,6)}$, there are also reports that NMES has no significant effect on the recovery of swallowing function ${ }^{7)}$. In addition, in retrospective studies, its treatment effect was significant only in mild and moderate dysphasia ${ }^{8}$.

To date, although domestic studies on dysphagia have mainly focused on its characteristics, only a few studies have compared the treatment effect of NMES using temperature-tactile stimulation, which is mainly used in clinical circumstances ${ }^{9}$.

This study compared NMES and thermal tactile oral stimulation (TTOS), a traditional swallowing recovery treatment, in patients with sub-acute dysphagia caused by stroke.

\section{SUBJECTS AND METHODS}

Subjects of the present study were 55 patients diagnosed with dysphagia caused by stroke in the rehabilitation departments of three general hospitals in Seoul and Inchon between September 2014 and January 2015 who agreed to participate in this study after understanding its contents. This study was approved by the Institutional Review Board of N University and was conducted in accordance with the ethical standards of the Declaration of Helsinki. As the minimum number of samples calculated based on power analysis was 53 with a significance level $(\alpha)=0.05$, effect size 0.5 , and power of test $(1-\beta)=0.95$ on the standard of $t$-distribution, the number of samples of this study was appropriate. The standards of selection for the subjects of this study were as follows: $<6$ months had passed since the dysphagia diagnosis was made; mild or moderate dysphasia; no mental illness such as depression; fourth, no nasogastric tube; fifth; and no cognitive disorder $>24$ points on the Korean mini-mental status exam.

All participants were randomly assigned into the NMES group $(n=27)$ or TTOS group $(n=28)$ using the table of random numbers. Subject characteristics are presented in Table 1.

The NMES group received 30 minutes of stimulation per day 5 days per week for 3 weeks by a swallowing therapist with Vitalstim certification using Vitalstim ${ }^{\circledR}$ (AP2116; DJO Company, USA) for a total of 15 treatments. Referring to Freed et al., the electrodes were attached to the mylohyoid and thyrohyoid muscles, the frequency of vibration was set at $80 \mathrm{~Hz}$ with a width of $300 \mathrm{~ms}$, and the wave pattern was bi-phasic ${ }^{5}$. The electric current intensity started at $2.5 \mathrm{~mA}$ and, set at a comfortable level for the patients by increases in $2.5-\mathrm{mA}$ increments, and had a maximum of $20.0 \mathrm{~mA}$. The subjects were instructed to perform dry swallowing during the electric stimulation.

The TTOS group repeated the 30-minute stimulation on the anterior faucial arch with an ice stick to facilitate pharyngeal swallowing each day 5 days per week for 3 weeks. The treatment consisted of rubbing the anterior faucial arch up and down 5 times, inducing dry swallowing, and repeating this method for 30 minutes.

Swallowing recovery was verified by a medical doctor of rehabilitation medicine who did not participate in the study using a videofluoroscopic swallowing study (VFSS) and Functional Dysphagia Scale based on videofluoroscopic studies (VFS) ${ }^{10)}$. For VFSS, this study used Multistar TOP (Siemens, Erlangen, Germany) and analyzed video data with VirtualDub v1.10.2 (VirtualDub, Korea) which is capable of analyzing 30 frames per second.

VFS is a scale that is used to identify overall swallowing problems such as aspiration observed in VFSS and consists of a total of 100 points. The higher the VFS score, the more severe the swallowing function problems. When VFS test was developed, its sensitivity was $81-82 \%$ and specificity was $70.7-92 \%{ }^{10}$ ).

This study had a nonequivalent control group pretest-posttest design. For the analysis, a preliminary homogeneity test was first conducted with an independent sample t-test or $\chi^{2}$ test, while a pre-post efficiency test was conducted using a paired-sample t-test. SPSS 21.0 (IBM Inc., Chicago, IL, USA) was used for the analysis, and the significance level was 0.05 .

\section{RESULTS}

In the test on homogeneity of age, VFS and outbreak duration of the NMES and TTOS groups with independent t-test and chi-square test, there was no significant difference in all items (Table 1).

The analysis of pre-post values of VFS of the NMES and TTOS groups using a paired t-test revealed no significant difference between the two groups despite both having decreased mean VFS values after treatment (Table 2). 
Table 1. Patient characteristics prior to the study

\begin{tabular}{lcc}
\hline Variable & NMES $(\mathrm{n}=27)$ & TTOS $(\mathrm{n}=18)$ \\
\hline Gender & & \\
Male & 17 & 16 \\
Female & 10 & 12 \\
Age (years) & $65.2 \pm 7.7$ & $67.5 \pm 8.3$ \\
Duration of dysphagia (months) & $4.5 \pm 1.1$ & $4.8 \pm 1.2$ \\
VFS (score) & $26.1 \pm 13.1$ & $25.5 \pm 11.5$ \\
\hline *p $<0.05$, mean \pm SD. & \\
NMES: neuromuscular electrical stimulation; TTOS: thermal tactile oral stimulation; \\
Functional Dysphagia Scale based on videofluoroscopic studies (VFS)
\end{tabular}

Table 2. VFS results of NMES and TTOS

\begin{tabular}{lcc}
\hline VFS & NMES $(\mathrm{n}=27)$ & TTOS $(\mathrm{n}=18)$ \\
\hline Pre-test & $26.1 \pm 13.1$ & $25.5 \pm 11.5$ \\
Post-test & $14.3 \pm 8.5$ & $15.8 \pm 9.2$ \\
\hline *p $<0.05$. & \\
VFS: videofluoroscopic & study; NMES: neuromuscular electrical \\
stimulation; TTOS: thermal tactile oral stimulation
\end{tabular}

\section{DISCUSSION}

The purpose of treating dysphagia in stroke patients is to reduce the risk of aspiration and enhance their ability to swallow food safely. Both treatment methods tested here significantly enhanced swallowing function. Multiple studies have reported that NMES enhanced swallowing function ${ }^{6,9,11)}$, perhaps more effectively than TTOS $^{5)}$, in the pharyngeal phase ${ }^{9)}$ and enhanced function in the oral phase ${ }^{12}$. In particular, Freed et al. recommended using NMES with complementary treatment and TTOS since they have limited effect in strengthening the swallowing muscles and enhancing coordination ${ }^{5)}$.

As in these preceding studies, 3-week NMES treatment in this study effectively enhanced swallowing function. NMES is a treatment method that recovers muscle function by emitting minute electricity on the skin, enhances and the larynx lifting motion, and ultimately recovers swallowing function ${ }^{13)}$. Thus, it is expected that applying NMES to patients with sub-acute dysphagia caused by stroke can enhance their swallowing function.

The second important discovery of this study was that the effect of NMES treatment on patients with sub-acute dysphagia did not have any significant difference from that of TTOS. Although treatments for dysphagia differ depending on its cause, complementary treatment, TTOS, and electric stimulation treatment are performed. Unlike complementary treatment and TTOS, which are covered by health insurance, NMES is excluded from coverage, making it expensive. Nevertheless, NMES treatment is not merely frequently conducted for sub-acute dysphagia but is performed by $72.3 \%$ of swallowing treatment experts ${ }^{14,15)}$. Therefore, it is necessary to prescribe guidelines for swallowing treatment considering the economic difficulties of patients with dysphagia; accordingly, future comparisons of the effectiveness and economic considerations of various swallowing treatments are required.

The limitations for this study are as follows. First, the treatment period was only 3 weeks long, so future studies are required to investigate the effect of swallowing rehabilitation based on treatment duration. Second, since the subjects were all diagnosed with sub-acute dysphagia, the study results cannot be generalized for all types of dysphagia. Thus, caution should be taken in the interpretation of the study results.

In this study, both NMES and TTOS significantly enhanced the swallowing function of patients with sub-acute dysphagia. To verify the effectiveness of NMES, future follow-up studies of patients with chronic dysphagia are required.

\section{REFERENCES}

1) Logemann JA: Evaluation and treatment of swallowing disorders. Austin: Pro-ed, 2002, pp 148-153.

2) Falsetti P, Acciai C, Palilla R, et al.: Oropharyngeal dysphagia after stroke: incidence, diagnosis, and clinical predictors in patients admitted to a neurorehabilitation unit. J Stroke Cerebrovasc Dis, 2009, 18: 329-335. [Medline] [CrossRef]

3) Martino R, Foley N, Bhogal S, et al.: Dysphagia after stroke: incidence, diagnosis, and pulmonary complications. Stroke, 2005, 36: 2756-2763. [Medline] [CrossRef] 
4) Hammond CA, Goldstein LB: Cough and aspiration of food and liquids due to oral-pharyngeal dysphagia: ACCP evidence-based clinical practice guidelines. CHEST J, 2006, 129: 154-168. [CrossRef]

5) Freed ML, Freed L, Chatburn RL, et al.: Electrical stimulation for swallowing disorders caused by stroke. Respir Care, 2001, 46: 466-474. [Medline]

6) Bülow M, Speyer R, Baijens L, et al.: Neuromuscular electrical stimulation (NMES) in stroke patients with oral and pharyngeal dysfunction. Dysphagia, 2008, 23: 302-309. [Medline] [CrossRef]

7) Kiger M, Brown CS, Watkins L: Dysphagia management: an analysis of patient outcomes using VitalStim therapy compared to traditional swallow therapy. Dysphagia, 2006, 21: 243-253. [Medline] [CrossRef]

8) Shaw GY, Sechtem PR, Searl J, et al.: Transcutaneous neuromuscular electrical stimulation (VitalStim) curative therapy for severe dysphagia: myth or reality? Ann Otol Rhinol Laryngol, 2007, 116: 36-44. [Medline] [CrossRef]

9) Jung BH: The effect of electrical stimulation for swallowing function in stroke patients with dysphagia. J Kor Soc Occup Ther, 2007, 15: 25-33.

10) Han TR, Back NJ, Park JW: The Functional dysphagia scale using videofluoroscopic swallowing study in stroke patients. J Korean Acad Rehabil Med, 1999, 23: $1118-1126$.

11) Kim SR, Kwon KH, Cho BJ: The effects of neuromuscular electrical stimulation on pharyngeal transit time. J Phys Ther Sci, 2013, 25: 849-851. [Medline] [CrossRef]

12) Park CL, O'Neill PA, Martin DF: A pilot exploratory study of oral electrical stimulation on swallow function following stroke: an innovative technique. Dysphagia, 1997, 12: 161-166. [Medline] [CrossRef]

13) Wang JS, Lee JH, Kim NJ: Effects of neuromuscular electrical stimulation on masticatory muscles in elderly stroke patients. J Phys Ther Sci, 2015, 27: 2767-2770. [Medline] [CrossRef]

14) Wu HS, Jang KH, Cha TH et al.: A study of the status of dysphagia rehabilitation conducted by occupational therapists in Korea. J Kor Soc Occup Ther, 17: $67-77$.

15) Moon TY, Kwon KH, Kim JW, et al.: A study of functional dysphagia scale improvements after neuromuscular electrical stimulation. J Phys Ther Sci, 2013, 25: 61-64. [CrossRef] 